\title{
All-optical networks provide flexibility for platforms on the Global Information Grid
}

Ketan Patel, Peter Guilfoyle, and Julian Cheng

A fault-tolerant, decentralized, all-optical network topology improves network performance compared to platforms based on electronic switches.

The advent of network-centric operations and the Global Information Grid (GIG) —which provides information storage, management, processing and transport for U.S. defense and security functions-have highlighted the need for ultra-wide bandwidth networks to efficiently and securely route multigigabit data streams among air, space, and ground platforms. Fiber optics (FO) plays an important role in the GIG: beyond the inherently higher bandwidth as compared to copper cables, the electromagnetic interference immunity and reduced weight of FO will be crucial. Conventional FO-based networks use a star topology in which all transmissions go through a central node and electronic switches dynamically route information based on header information embedded in the data streams. A decentralized network, however, enhances the GIG by enabling asymmetric links as well as broadcast and point-to-point high rate data communication links. These are particularly useful when latency must be managed for mission critical links.

OptiComp Corp. (OCC) is developing a new optoelectronics technology platform that can be used in conjunction with an all-optical routing paradigm to significantly improve network system performance as compared to platforms based on electronic switches, especially at multi-Gigabits per second (Gbps) data rates. The very high level of interconnectivity offered by the OCC switch architecture provides multiple paths among the network nodes, thus allowing a relatively simple architecture to achieve exceptional levels of performance and reliability. The paradigm most comparable to this network is the highly successful 'ThinNet' (i.e., 10BASE2 Ethernet), which uses the Carrier Sense Multiple Access / Collision Detect (CSMA/CD) protocol and destination address headers in each data packet

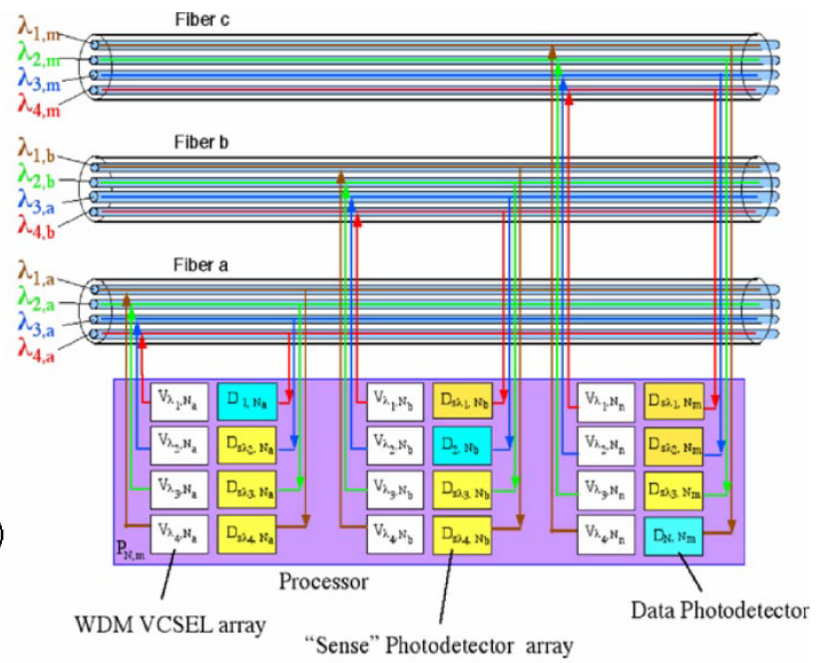

(b)

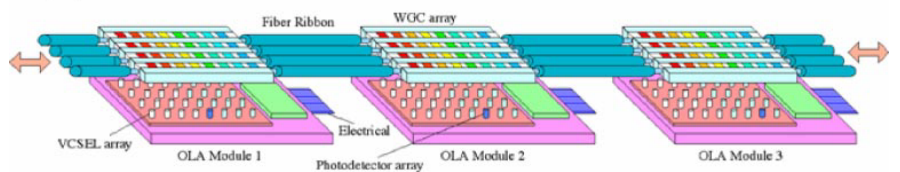

Figure 1. The parallel fiber wavelength division multiplexing (WDM) $N^{4}$ crossbar module utilizes 2D arrays of monolithically integrated coarse WDM vertical-cavity surface-emitting lasers (VCSELs) and resonant cavity detectors.

message. The 10BASE2 Ethernet uses electrical taps or 'tees' to communicate over a shared transmission medium in a switchless environment without the use of central arbitration, access tokens, or assigned time slots to indicate when a station will be allowed to transmit.

Envisioned for use with OCC's optoelectronics switch is an $\mathrm{N}^{4}$ crossbar module, shown in Figure 1, in which each of $\mathrm{N}$ nodes can directly transmit or receive data on any of $\mathrm{N}$ waveguide or fiber channels via an N-element array of wavelength-selective

Continued on next page 
(a)

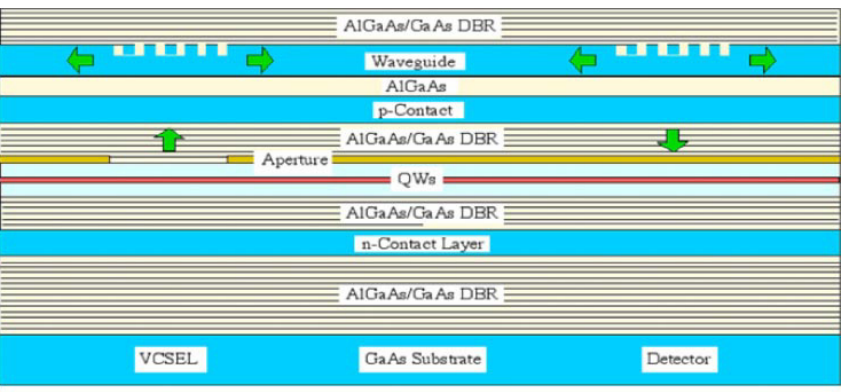

(b)

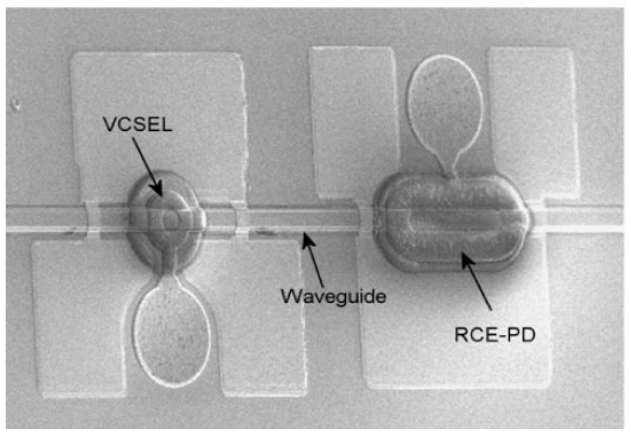

Figure 2. (a) This cross-section of the distributed switching module parallel to the waveguide shows the monolithically integrated gratings for multiplexing and demultiplexing the optical signals. $Q W=$ quantum wells; $D B R=$ distributed Bragg reflector. (b) Scanning electron micrograph of a fabricated VCSEL and RCEPD with embedded waveguide/grating structure.

vertical-cavity surface-emitting laser (VCSEL)/detector pairs that 'tap' into the fiber. With $\mathrm{N}$ parallel fibers and $\mathrm{N}$ wavelength channels on each fiber, each node has a total of $\mathrm{N}^{2}$ access ports. Communication between nodes utilizes the same CSMA/CD protocol used in 10BASE2 Ethernet systems but, critically, each node now has $\mathrm{N}^{2}$ possible channels to communicate over. In the $\mathrm{N}^{4}$ architecture, power sensing by the photodetectors determines the availability of all combinations of fiber and wavelength channels. When data is ready to be transmitted, the transmitting node attempts to handshake with the target node by systematically cycling through each 'dark' channel until the destination node responds. This configuration results in a highly fault tolerant, locally autonomous routing system that does not require a central network controller.

Optical interconnections in the $\mathrm{N}^{4}$ architecture are still based on the use of the optical tees, which are now configured in a dense, 2D, integrated format. OCC's interconnect technology uses integrated linear arrays of vertical-cavity lasers and photodetectors that are optically interconnected laterally by an embedded waveguide: light is coupled to it by a unique surface-normal coupling technique. The resulting compact optoelectronic module thus consists of low-power, integrated, waveguide-coupled $\mathrm{N} \times \mathrm{N}$ VCSEL and photodetector arrays.
Whereas conventional approaches ${ }^{1,2}$ to surface-normal waveguide coupling by means of volume gratings have resulted in 60-70\% efficiency, OCC's technology can achieve nearly 100\% coupling of the VCSEL emission into the waveguide by directly embedding the diffraction grating and waveguide within the VCSEL cavity. Figure 2 shows a schematic cross-section and a scanning electron micrograph of the fabricated devices in a typical integrated optoelectronic array depicted parallel to the waveguide, thus illustrating the epitaxial growth sequence for the monolithic structure. Likewise, a resonance cavity-enhanced photodetector (RCEPD) is used; embedding in its cavity an optical waveguide and a grating coupler enables an efficient and compact optical tap.

System analysis of $1 \mathrm{Gbps}$ communications links with cascaded optical tees shows that, with proper management of link losses and grating-coupling efficiencies, as many as 5-6 nodes can be linked serially. Higher levels of optoelectronic integration, such as the incorporation of optical amplifiers, can further extend the range of the $\mathrm{N}^{4}$ architecture to 10 nodes or more per serial link.

Certainly, many solutions can provide a reconfigurable, highly flexible and robust network. Conventional technologies, however, do not lend themselves to compact, high-performance broadband network solutions that can be deployed on a variety of small platforms that are size, weight, and power critical. The technologies that we are developing, however, not only enable novel survivable network solutions but also the means to achieve reconfigurability with minimal impact on size, weight and cost.

\section{Author Information}

\author{
Ketan Patel, Peter Guilfoyle, and Julian Cheng \\ OptiComp Corporation \\ Zephyr Cove, NV
}

Dr. Ketan M. Patel is member of technical staff at OptiComp Corp. He previously was a member of the technical staff in the gallium arsenide foundry at Texas Instruments in Dallas, Texas. His research interest is in integration of photonic and microwave circuits.

Peter Guilfoyle, founder, Chairman of the Board, and Chief Executive Officer of OptiComp Corp, has been a prime federal contractor since 1985. He has developed a second generation, 32bit, digital optoelectronic computer (DOC II) as well as a High Performance Optoelectronic Computing (HPOC) module sys- 
tem architecture utilizing global (N4), free-space 'smart' optical interconnects.

Dr. Julian Cheng has more than 25 years of experience in optoelectronics and fiber optic communications technology, with more than 11 years at AT\&T Bell Laboratories, both as a Distinguished Member of Technical Staff and as manager in the Lightwave Components Division. As a researcher at Bell Labs, he made contributions in the area of InP-based optoelectronic devices, optical datalinks and the first parallel fiber optic data link technology.

\section{References}

1. S. M. Schultz, E. N. Glytsis, and T. K. Gaylord, Volume grating preferential-order focusing waveguide coupler, Opt. Lett. 24, pp. 1708-1710,1999.

2. D. Taillaert, W. Bogaerts, R. Bienstman, T. F. Krauss, P. Van Daele, I. Moerman, S. Verstuyft, K. De Mesel, and R. Baets, An Out-of-Plane Grating Coupler for Efficient Butt-Coupling Between Compact Planar Waveguides and Single-Mode Fibers, J. Quantum Elect. 38, pp. 949-954, 2002.

3. R. E. Shotwell ed., The Ethernet Sourcebook, North-Holland, New York, 1985. 